\title{
The Research of Wavelet Transform Blind Equalization Algorithm Based on Ant Colony Optimization
}

\author{
Lei Jinhui ${ }^{1}$ and Li Jingli ${ }^{2}$ \\ ${ }^{1}$ School of Information Engineering, Henan Institute of Science and Technology, \\ Henan Xinxiang, China \\ ${ }^{2}$ Department of Automation Engineering, Yellow River Conservancy Technical \\ Institute, Henan Zhengzhou, China \\ 132745994@qq.com, ${ }^{2} j$ ingli613@163.com
}

\begin{abstract}
ACA (Ant Colony Algorithm) is a smart global random search algorithm, which is combined with blind equalization algorithm. In optimizing and initializing equalizer weight vector, ACA can avoid falling into local extremum easily in Stochastic Gradient Descent Algorithm of CMA and improve the convergence speed and reduce the steady-state mean square error greatly. This paper mainly studies the ACA to initialize the equalizer weight vector and the theory of wavelet transform, comes up with the wavelet blind equalization algorithm based on Ant Colony Optimization (ACA-WTCMA).The simulation results of underwater acoustic channel verify the effectiveness of the algorithm.
\end{abstract}

Keywords: Ant Colony Algorithm, Wavelet Transform (WT)

\section{Introduction}

Ant colony algorithm is a novel simulated evolutionary algorithm, which is proposed recently. In the algorithm, the feasible solution will eventually approach the optimal solution with the greatest probability after several times of iterative. Ant colony algorithm was first proposed by Italy scholars M. Dorigo, V. Maniezzo etc at the beginning of the 90 's of twentieth century. This method is used to solve the problems of traveling salesman, assignment problem, job-shop scheduling problem and so on. And, it got a series of good experiment results. Thus, ant colony algorithm has caused other researchers' attention, and uses the method to solve some practical problems. Although this method just started, but these preliminary studies have shown some superiority of the ant colony algorithm in solving the complex optimization problem, prove that it is a promising method.

Blind equalization which doesn't need sequence training can effectively eliminate the intersymbol interference in underwater acoustic channel, thus save bandwidth and improve efficiency of communication. ACA is a smart global random search algorithm, which is combined with blind equalization algorithm. In optimizing and initializing equalizer weight vector, ACA can avoid falling into local extremum easily in Stochastic Gradient Descent Algorithm of CMA and improve the convergence speed and reduce the steady-state mean square error greatly. 


\section{Ant Colony Algorithm: Principle Analysis \& Algorithm Description}

\subsection{Information System of Ants}

Both the morphology and behavior of ants is quite simple. An ant can only make no more than fifty movements, most of which are used to pass on information. However, an ant colony, composed of such simple ant individuals, is analogous to a highly-structured social organization. In most cases, ant colonies are capable of fulfilling far more complicated tasks which is beyond the ability of an ant itself. Each individual of an ant colony takes charge of different work, and as a group, they can successfully fulfill their tasks [1]. Ants, as one kind of the social insects, also communicate and pass on information amongst each other despite the labor division. An ant colony has a unique information system, including visual signals, voice communications, and pheromones. It is the information system that enables ants to crowd around a piece of candy [2].

An ant colony has a fantastic information system, including visual signals, voice communications, and unique silent language. Ants communicate with each other using various combinations of chemical substances, touch, and body actions. The unique ability of ants to control the surrounding environment has been acquired from the constant evolvement of their advanced social behavior.

Ant behavior is subject to pheromones to a large degree. An ant colony is divided into two classes: the queen and worker ants. The queen secretes a type of pheromone called "queen substance" to control the growth of workers. Pheromones can be used to ask for or exchange trophic eggs and special secretions [3]. An alarm pheromone will send ants into attack frenzy and encourage them to do something as planned. In addition to this, ants use pheromones to identify themselves from each other; an ant could not survive without the pheromone of its ant colony.

\subsection{Heredity and Evolvement of Ant Colonies}

Genetics provides researchers with the access to the study on the mutual cooperation and altruism of ant colonies. The sexual reproduction of ants is slightly different from that of diplonts, because ants belong to haplodiploid insects. The fertilized eggs laid by the queen grow into worker ants or new queens, while the unfertilized eggs become male ants. Males are haploid, while females (workers and the queen) are diploid. Based on that, the coefficient of kinship between workers (full sisters) is 0.75 rather than 0.5. This is because half of an ant's genes are the same as its haploid father, but the other half of its genes are inherited from only one half of the genes of its diploid mother. Therefore, for ants, sisterhood is more important than the mother-daughter relationship. It thus seems that cooperative behavior and altruism ought to be fully developed in a family that has closest relationships [4].

Generally speaking, ants exist as a whole; they do not survive for the sake of just an individual. It is the highly evolved social adaptiveness that enables the age-old, small ants to occupy a place in the natural world and reproduce to this day. This is why they are praised as "the flowers of wisdom".

\subsection{Foraging Behavior \& Strategies of Ants}

Foraging behavior of ant colonies is import and interesting. Based on the observation and researches of entomologists, ants in the natural world are able to find the shortest route between their nest and the source of food even in the dark. Moreover, they can find a new route if the environment changes. 
Ants secrete a chemical substance on the way back to their nest after food foraging; it is known as pheromone (also called ectohormone). In this way, ants have their own pheromone trails. Other ants nearby perceive the substance and determine its intensity, so that it can find the right direction toward the trail that has high pheromone density. Pheromone trails help ants to find the way back to the source of food (or nest); other ants may also follow the trail and then find the source of food.

Most ants leave traces and follow traces in the course of food foraging. They secrete pheromones on their way back to the nest from a source of food. Other ants may follow the trace to find the food source. "Conscription" refers to the process while an ant is heading toward a source of food under the influence of other ants or the pheromone trails. The "conscription" that merely depends on chemical traces is called "large-scale conscription". As a matter of fact, the movements of ants are coordinated based on the information they have obtained; they are divided into different groups so that they can support each other. When the number of ants reaches the critical value, some well-organized ant armies begin to emerge. The foraging behavior of ants turns out to be a type of self-organization behavior. Ants choose the way to a source of food by themselves, thus, the foraging behavior of ants is considered to be a self-organization behavior.

\section{Basic Principle and Model of Ant Colony Algorithm}

As social animals, ants have rather simple individual behavior, but ant colonies that are composed of simple individuals show pretty complicated behavior characteristics. This is because ants emit pheromones on the path to a source of food, enabling the nearby ants to move toward the trail that has high pheromone density. The collective behavior of ants manifests itself as a positive feedback phenomenon, and the ant's behavior of following a trail is called "autocatalytic behavior". Since its principle is on the basis of a positive feedback system, the behavior of ant colonies may also be regarded as a Reinforcement Learning System [5].

The principle of ant colony algorithm comes from entomologists' observation: when looking for food, ants can release a specific secretions-pheromone in the path, which influences nearby ants' behavior. When more ants pass the path, such information is also accumulated. Later, the probability of choosing the path is higher, which increases the attraction of the path. Based on the internal biological synergy mechanism, Ant colony gradually formed the shortest route unconsciously. This model enlightens ACA and ACA is used to solve optimization problems. Every solution of optimization problems in ACA is an ant in the search space, and every ant has a fitness value (in proportion with the pheromone to be released) determined by optimal function. Ant colony algorithm is linked together with solving TSP problems [6]. We now use the following figures to explain the basic idea of ACA. (The number of ants is 30 ) 


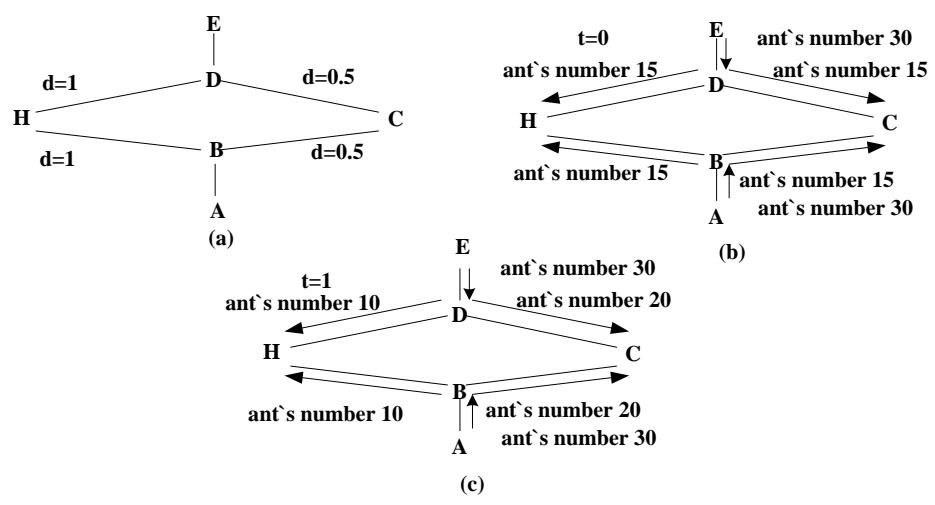

Figure 1. Basic principle and model of ant colony algorithm

We assume that the distance between $\mathrm{D}$ and $\mathrm{H}, \mathrm{B}$ and $\mathrm{H}$ as well as $\mathrm{B}$ and $\mathrm{D}$ (through $\mathrm{C}$ ) is 1.C is located in center of the path from D to B (In Figure 1a).Then, we consider the situations of Ant colony system at discrete time points $(t=0,1,2 \ldots)$. In every unit of time, we assume that there are 30 ants from $\mathrm{A}$ to $\mathrm{B}$, the other 30 ants from $\mathrm{E}$ to $\mathrm{D}$, and the walking speed of both is 1 (the walking distance in a unit of time is 1 .when they walking, the concentration of pheromone an ant leaves is $\mathrm{I}$. At the time of $\mathrm{t}=0$, there is not any pheromone, but there are 30 ants at $\mathrm{B}, 30$ ants at $\mathrm{D}$, waiting for departure. They choose the path completely at random; therefore ants at both points walk in two directions. As a result, 15 ants choose the left path $\mathrm{D}-\mathrm{H}$, left $\mathrm{B}-\mathrm{H}$; another 15 ants take the right path D-C, B-C. In the moving process, these ants left pheromone. After one second, 15 ants of the 30 ants started from point $\mathrm{D}$ arrived at the $\mathrm{H}$ point, 15 ants reached $\mathrm{B}$ through $\mathrm{C}$. The same process goes on to the ants at B. Obviously, in the same time there are 15 ants went through and left pheromone on path D-H-B, while 30 ants left pheromone on path D-C-B. The pheromone strength of path D-C-B is two times of the D-H-B path. Therefore, when the 30 ants were back to A, E to choose path, they will be more likely to choose the path of D-C-B, two times of the D-H-B path. Therefore, the number of ants on D-H-B changes into 10, half of the number on D-C-B. The path that is shorter is quickly strengthened by pheromone, and its advantage is found by ant colony very soon.

In this way, we can understand the basic idea of ACA: If at a given point, an ant need to choose paths. The paths that are more preferred by the former ants are more likely to be selected by the latter ants. More pheromone means the path is shorter, which means a better solution. ACA has the following advantages:

a). ACA is a distributed parallel algorithm. The search process of each ant is independent, which tends to fall into local optimum. But information exchange and transfer between individuals constantly can help to find better solution.

b). ACA is a kind of positive feedback algorithm. The higher the pheromone level is, the more ants the path will attract, which increases the pheromone levels. Thus the evolution process of the algorithm speeds up.

c). ACA has strong robustness. If we modify the model slightly, it can be applied to solve other problems. 


\section{Basic Theories about Wavelet Transform}

The traditional signal analysis is based on Fourier transform. Fourier analysis focuses on either time domain or frequency domain, making it unable to describe the time-frequency localization properties of signals. Nonetheless, such properties are the most fundamental and important for non-stationary signals [7].

Short-Time Fourier Transform (STFT) falls under the category of Windowed Fourier Transform. The basic idea of STFT is as follows. Assume the non-stationary signal $\mathrm{f}(\mathrm{t})$ remains stable (pseudo-stable) within a short time interval while analyzing the window function $\mathrm{g}(\mathrm{t})$; analyze the window function and make sure $\mathrm{f}(\mathrm{t}) \mathrm{g}(\mathrm{t}-\mathrm{t} 0)$ is a stationary signal in different finite time width, and then calculate the power spectrum at different time. STFT, as a matter of fact, is a signal analysis method based on single resolution and a fixed short-time window function. It can represent the local frequency characteristics of signals along with the sliding of the window function. However, STFT must choose another window function $\mathrm{g}(\mathrm{t})$ to change the resolution. Thus, STFT is capable of analyzing stationary signals, but it cannot process non-stationary signals [8]. In the case of quick changes in signal waveforms, high frequency becomes dominant, and high time resolution is needed; in the case of slow changes in signal waveforms, low frequency is the dominant frequency, so high frequency resolution is required. Obviously, the sliding window method that has equivalent time domains is not applicable to all signals.

Wavelet transform is a multiresolution analysis method with the purpose of "seeing both the forest (the general view of a signal) and the trees (signal details)". It is capable of characterizing local features of signals in both the time domain and the frequency domain. It has high frequency resolution and low time resolution in the low frequency part; but in the high frequency part, it has high time resolution and low frequency resolution. It is a timefrequency localization analysis method, which has fixed window size (window area) but changeable shape, time window, and frequency window. For this reason, wavelet transform is known as the mathematical microscope for signal analysis [9].

Wavelet, briefly, is a family of functions $\varphi_{a, b}(t)=|a|^{\frac{1}{2}} \varphi\left(\frac{t-b}{a}\right), a, b \in R, a \neq 0$ as a result of translation and scaling of the function that meet the condition--- $\int \varphi(t) d t=0$; Given a signal of finite energy $\int \varphi(t) d t=0$, i.e., $f(t) \in L^{2}(R)$, the Continuous Wavelet Transform (CWT) is defined as follows[10]:

$W_{f}(a, b)=\int f(t) \varphi_{a, b} *(t) d t \quad a \neq 0$

$\varphi^{*}(t)$ is the conjugate of the wavelet function $\varphi(t)$.

If $\varphi(t)$ meets the condition below:

$$
C_{\varphi}=\int_{-\infty}^{+\infty} \frac{|\hat{\varphi}(\omega)|^{2}}{|\omega|} d \omega<+\infty
$$

It can be ideally restored:

$$
f(t)=\frac{1}{C_{\varphi}} \iint_{R} \frac{W_{f}(a, b) \varphi_{a, b}^{*}(t)}{a^{2}} d a d b
$$

In practical applications, especially in the computer field, CWT should be processed with discretization. The relevant discrete wavelet is now defined as:

$$
C_{m, n}(f)=\int_{-\infty}^{+\infty} f(t) \varphi_{m, n}^{*}(t) d t
$$


Where $\varphi_{m, n}(t)=|a|^{-\frac{m}{2}} \varphi\left(a_{0}^{-m} t-n b_{0}\right)$.

Below is the physical significance of wavelets from the perspective of signals and systems.

Based on $\int_{R} \varphi(t) d t=0, W_{f}(a, b)$ can be described as the smoothing left by a signal (function) $f(t) \in L^{2}(R)$ after passing through a band-pass filter. Another definition of wavelet transform is introduced to explain the relationship between wavelet transform and coefficients more clearly.

$$
W_{f}(s, t)=f(t) * \varphi_{s}(t)=\frac{1}{s} \int_{R} f(u) \varphi\left[\frac{t-u}{s}\right] d u
$$

Where $\varphi_{s}(t)=\frac{1}{s} \varphi\left[\frac{t}{s}\right], s \neq 0$. In fact, the two definitions mentioned above are equivalent.

Thus, wavelet transform can be deemed as the response in the system $\varphi_{s}(t)$ after the signal $f(t)$ is inputted. And $\varphi_{s}(t)$ is the response of the system to the function $\delta$, that is, $W_{\delta}(s, t)=\varphi_{s}(t)$. Thereby, the process of wavelet transform can be regarded as the smoothing process.

Based on the above analysis, wavelet transform is the development of Fourier transform. Its structure and results supplement each other, and they both depend on Fourier analysis. There are several differences between wavelet transform and Fourier transform [11]:

(1) Fourier transform, in essence, resolves the signal of finite energy $f(t)$ into the space with $\left\{e^{j w t}\right\}$ as the orthogonal basis. But the essence of wavelet transform is to resolving the signal of finite energy $f(t)$ into the space composed of both $W_{-j}(j=1,2, \cdots, J)$ and $V_{-J}$.

(2) The basic functions Fourier transform employs $\operatorname{are} \sin (\omega t), \cos (\omega t), \exp (\omega t)$; they are all unique. But the functions used by wavelet transform (i.e., wavelet function) are not exclusive. For the same engineering problem, the results sometimes differ significantly from each other if it is analyzed with different wavelet functions. How to select the proper wavelet function is a quite difficult problem in the actual application of wavelet analysis. At the present time, researchers tend to choose a wavelet function on the basis of experience or trial and error (contrast analysis of results).

(3) Fourier transform has good localization ability in the frequency domain, especially for the stationary signals that contain simple frequency components. It is easier for Fourier transform to describe a signal into the superposition of all frequency components such as $\sin \left(\omega_{1} t\right)+0.345 \sin \left(\omega_{2} t\right)+4.23 \cos \left(\omega_{3} t\right)$. But in the time domain, Fourier transform does not have the localization ability, meaning it is impossible to see the form of $f(t)$ at random time from $F(\omega)$, the Fourier transform of the signal $f(t)$. Actually, $F(\omega) d \omega$ is the amplitude of the harmonic component at the frequency of $\omega$, which is determined by the overall form of $f(t)$ in the Fourier expansion.

(4) The bigger the scale $a$ is in wavelet analysis, the smaller $\omega$ is in Fourier transform.

(5) In STFT, the transformation coefficient $G(\omega, \tau)$ mainly depends on the situation of signals during $[\tau-\delta, \tau+\delta]$. The time width is $2 \delta$ ( $\delta$ is only determined by the window function $g(t)$, so $2 \delta$ is a definite value). In wavelet transform, the transformation coefficient $W_{f}(a, b)$ mainly depends on the situation of signals during $[b-a \Delta \varphi, b+a \Delta \varphi]$. The 
time width is $2 a \Delta \varphi$, and it varies with the scale $a$. Therefore, wavelet transform has the ability of time-localization analysis.

(6) Based on the explanation of signals passing a filter, the difference between wavelet transform and STFT is as follows. The bandwidth of band-pass filters $\Delta f$ is irrelevant to the center frequency $f$ in STFT. On the contrary, the bandwidth of band-pass filters in wavelet transform $\Delta f$ is in direct proportion to the center frequency $f$, i.e., $Q=\frac{\Delta f}{f}=C ; \mathrm{C}$ is a constant. In other words, the filter has a relatively constant bandwidth known as equivalent Q-structure.

\section{Blind Equalization Algorithm based on ACA}

In blind equalization algorithm, CMA use cost function to get the gradient from equalizer weight vector, and get the weight vector's iteration equation. This stochastic gradient descent algorithm is lack of global search ability. We can use it to minimize the cost function, update the weight vector of equalizer, and seek the extremum of cost function. The cost function needs to be continuous and derivative. This algorithm has the advantages of simple structure, high stability and low computation. As well as it is easy to fall into local minima and make bigger error, the convergence speed is slow.

ACA is a global random search algorithm. By updating particle's position and velocity iteratively, it ensures that the algorithm converges to the optimal position of a particle and avoids falling into local optima. Therefore, bring ACA into CMA can use the ACA's global search to find the optimal initial weight vector, which can make up for the defects in CMA of falling into extremum.

\subsection{Description of ACA-CMA}

\section{(1)Basic idea}

First, initialize a set of weight vectors randomly, and consider this group of weight vector as decision variable of ACA. Then, set the input signal of ACA as the equalizer's input signal together with the cost function of CMA to determine the fitness function of ACA and consider the fitness function as the cost function of the blind equalizer. Through iteration, we can find the weight vector when the fitness function gets its optimum, and consider the weight vector as the initial weight vector of CMA.

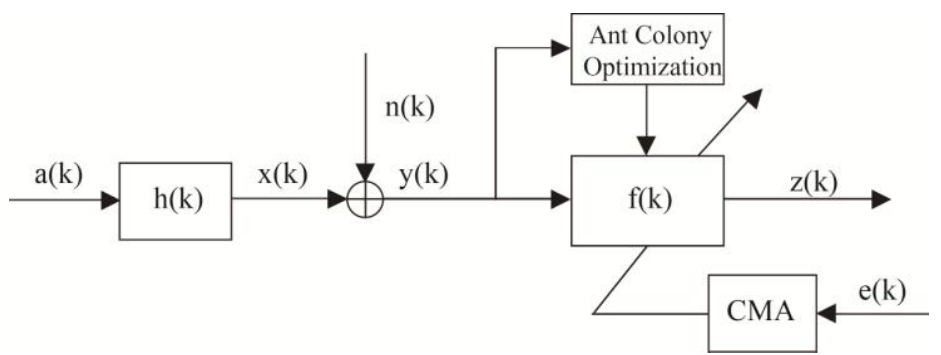

Figure 2. The schematic diagram of blind equalization based on Ant Colony Optimization

In Figure 2, a (k) is zero mean independent and identically distributed transmit signals; $h(k)$ is impulse response vector of the channel, and its length is $\mathrm{M}$; vector $\mathrm{n}(\mathrm{k})$ is additive white 
Gauss noise; vector ${ }^{y(k)}$ is the input signal and coefficient vector of equalizer, and its length is $2 \mathrm{~L}+1$, namely $f(k)=\left[f_{-L}, \cdots, f_{-1}(k), f_{0}(k), f_{1}(k), \cdots, f_{L}(k)\right]^{T}$ (the superscript $\mathrm{T}$ means transposing); ${ }^{z(k)}$ is the output signal of equalizer.

Assuming $a(k)=[a(k), \cdots, a(k-M+1)]^{T}, y(k)=[y(k+L), \cdots, y(k), \cdots, y(k-L)]^{T}$, reach the following equation by Fig.2

$$
y(k)=\sum_{j=0}^{M-1} h_{j} a(k-j)+n(k)=h^{T} a(k)+n(k)
$$

Output of equalizer: $\quad z(k)=y^{T}(k) f(k)$

Error of equalizer: $\quad e(k)=R_{C M}-|z(k)|^{2}$

CMA's cost function is

$$
\begin{aligned}
& J=E\left[e^{2}(k)\right]=E\left\{\left[z(k)-R_{C M}\right]^{2}\right\} \\
& \quad\left(R_{C M}=E\left\{|a(k)|^{4}\right\} / E\left\{|a(k)|^{2}\right\}\right. \text { is the of model value of transmitting }
\end{aligned}
$$

The iterative formula of weight vector is

$$
f(k+1)=f(k)-\mu z(k)\left(|z(k)|^{2}-R_{C M}\right) y^{*}(k)
$$

( $\mu$ is step length)

Equation (7) to (11) consist CMA, using ACA to search the weight vector of optimal initial equalizer.

\section{(2) The determination of fitness function}

CMA's cost function is

$$
J_{C M A}=\left(|z(k)|^{2}-R_{C M}\right)^{2}
$$

Thus, applying ACA to blind equalization algorithm needs to structure appropriate cost function algorithm. Since ACA is in the search space, and find the optimal fitness value through iteration. So adopt

$$
F\left(f_{1}\right)=1 / J\left(f_{i}\right), i=1,2, \cdots, M
$$

The formula (13) is ACA's fitness function. In the formula, $J\left(f_{i}\right)$ is the cost function of equalizer, $f_{i}$ is the position vector of particles ACA generated, namely the weight vector of equalizer.

\section{(3) The choice of optimal weight vector}

In ACA, each particle represents a potential solution in extremal optimization; each particle's fitness value is calculated by the fitness function. In this algorithm, the fitness function is determined by the reciprocal of cost function in the blind equalization algorithm. Because the cost function's minimum is found by adjusting the weight vectors of the equalizer in blind equalization algorithm, while ACA is used to search the maximum value point of fitness value to determine the optimal position vector of all the particles corresponding to fitness value. So, the reciprocal of the particle's fitness function is settled as the cost function of the algorithm. Through ACA optimization iteration, and compare the next iteration of the new particle fitness value and individual's extremum, fitness value of global 
extremum, update individual extremum and position vector of global extremum, find the weight vector(in the global optimal position) corresponding to the maximum fitness value, and see it as the initial weight vector of ACA-CMA.

\section{(4) The choice of iterating step size in algorithm}

Blind equalization algorithm is a kind of adaptive filtering algorithm in nature. By using some statistical properties of random signal, and extract the useful signal from interference signal, the output performance of adaptive equalizer can achieve the best according to the performance criterion of self-adaptive system .And, the best filter criteria governs the optimal parameters of signals with some characteristics. In the blind equalization algorithm, when other factor are the same, iterative step is to the key to control the speed of algorithm convergence and decide whether the algorithm can converge at the best point. When factors allow to astringe, the smaller the step length is, the slower the convergence speed will be, but the intersymbol interference is small. When the step length is larger, the convergence speed can be accelerated, but intersymbol interference will be larger. Therefore, the algorithm uses the theory of weighted vector's convergence in the minimum mean square algorithm (LMS), which select appropriate step factor to meet the criteria of weighted vector's convergence. Through the above analysis, after the convergence of the algorithm, a good blind equalizer should shorten the step length to reduce intersymbol interference and perform evenly.

\subsection{Wavelet blind equalization algorithm based on ACO}

In blind equalization algorithm, the main factor that affects the blind equalization algorithm's convergence speed is the auto-correlation matrix of the input signal. However, using the wavelet transform theory to reduce the self-correlation of input signals can accelerate the convergence of the algorithm; combine CMA with the ACA to come up with ACA-WT-CMA.

\subsubsection{The description of ACA-WT-CMA}

ACA finds the optimal weight vector of equalizer through iteration, while the cost function of WT-CMA seeks the gradient from the weight vector. The latter's the vector iteration equation to seek optimal is lack of global search ability. Through ACA's optimization iteration, can quickly find the weight vector corresponding to the maximum fitness value, and see it as the initial weight vector of ACA-CMA.

The schematic diagram of ACA-WT-CMA (Figure 3)

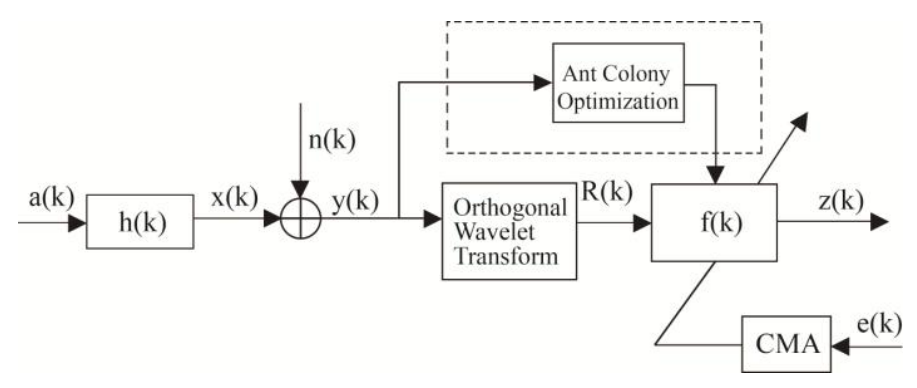

Figure 3. The schematic diagram of orthogonal wavelet transform blind equalization based on ACA 
By the theory of wavelet analysis and the equalizer weight vector formula,

$$
\begin{aligned}
& R(k)=y(k) Q \\
& z(k)=f^{T}(k) R(k) \\
& e(k)=R_{C M}^{2}-|z(k)|^{2} \\
& f(k+1)=f(k)+\mu \hat{R}^{-1}(k) z(k)\left(|z(k)|^{2}-R_{C M}^{2}\right) R^{*}(k)
\end{aligned}
$$

$R(k)$ is the signal vector, which is got after $y(k)$, orthogonal wavelet transform, $Q$ is orthogonal wavelet transform matrix, $R_{C M}^{2}=E\left\{|a(k)|^{4}\right\} / E\left\{|a(k)|^{2}\right\}$ is statistical model value of transmitting signal, $\quad \mu \quad$ is step length, $\hat{R}^{-1}(k)=\operatorname{diag}\left[\sigma_{1,0}^{2}(k), \sigma_{1,1}^{2}(k), \cdots, \sigma_{1, k_{l}-1}^{2}(k), \sigma_{I+1,0}^{2}(k), \cdots, \sigma_{I+1, k_{l}=1}^{2}(k)\right]$, 且 $\sigma_{i, n}^{2}(k)$ is the estimation of average power of $r_{i, n}(k)$ (wavelet transform coefficient), $\sigma_{I+1, n}^{2}(k)$ is the estimation of average power of $s_{I, n}(k)$ (scale transforming coefficient), their iteration formula are

$$
\begin{aligned}
& \hat{\sigma}_{i, n}^{2}(k+1)=\beta \hat{\sigma}_{i, n}^{2}(k)+(1-\beta)\left|r_{i, n}(k)\right|^{2} \\
& \hat{\sigma}_{i+1, n}^{2}(k+1)=\beta \hat{\sigma}_{i+1, n}^{2}(k)+(1-\beta)\left|s_{i, n}(k)\right|^{2}
\end{aligned}
$$

In the above formula, $\beta$ is smooth factor, and $0<\beta<1$. Formula(14) $\sim$ Formula(19) consist orthogonal wavelet transform's blind equalization algorithm(WT-CMA).

\subsubsection{The analysis of computational complexity}

Set the target vector dimension as $D$, ant colony as $N$, colony's max number of iteration as $M$. Then the initial ant colony's calculation complexity is $O(2 N D)$, the calculation complexity of each colony's fitness value is $O\left(N^{2}\right)$, the calculation complexity of the colony's updating is $O(2 N M)$. So, in one iteration, the total computational complexity is $O\left(2 N D+N^{2}+2 N M\right)$ at most. Only the size of particle and the max number of iterations can affect the complexity, and the calculation is relatively small. When $Q$ is known, the number of multiplications the weight coefficient needs to update for once is its computational complexity. In each weight coefficient's iterative process, WT-CMA needs to run $L$ point of orthogonal wavelet transform of signal $X(k)$ ( $L$ is for the length of the equalizer).According to the wavelet analysis theory, orthogonal wavelet transform's matrix --- $Q$ is equal to $L \times L$. Therefore, Formula (14) gets WT-CMA's computational complexity --- $O\left(L^{2}\right)$ In practice, $Q$ is sparse matrix. Assuming that the number of nonzero element in $Q$ 's each row is $H$, the computational complexity of formula (7) is $O(H L)$. By formula (14) (15) (16) and (13), the calculation complexity that WT-CMA updates weight coefficient once is $O(5 L+H L)$. By the iterative formula of CMA's weight vector's, the calculation complexity that CMA updates weight coefficient once is $O(5 L)$ From the above analysis, the 
computational complexity of ACA-WTCMA is $O\left(2 N D+N^{2}+2 N M+5 L+H L\right)$. Since the colony size is usually small, the increased computation that caused by the size is not large. Obviously, the increase of ACA-WT-CMA's calculation is mainly caused by ACA.

\subsubsection{Simulation experiment}

In order to test the validity of the ACA-WT-CMA and compare it with CMA, ACA-CMA, the author did the following simulation experiment.

Experiment 1: The mixed phase of underwater acoustic channel is $C=[0.3132-0.1040$ $0.89080 .3134]$; the launch signal is 8PSK, the length of equalizer is 16 , the signal noise ratio(SNR) is $20 \mathrm{~dB}$, the step lengths are $\mu_{C M A}=0.0009 、 \mu_{W T-C M A}=0.0025 、 \mu_{P S O-C M A}=0.00022$ 、 $\mu_{P S O-W T-C M A}=0.0005$ respectively; in CMA and WT-CMA, the fourth tap coefficient is set as 1 , the remaining are 0; using DB2 orthogonal wavelet to decompose the input signal of the channel ,decomposition level---2 layer, forgetting factor $\beta=0.999$, initial power value is set as 4;results of 20 times Monte Kano simulation as shown in Figure 4.

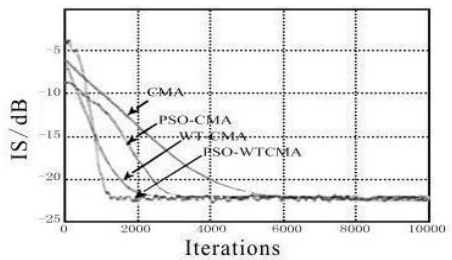

(a) Curves of ISI

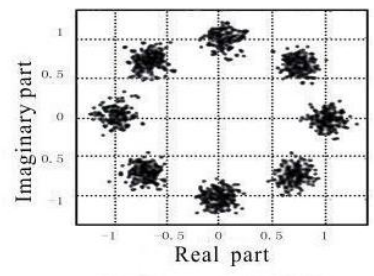

(c) Output of PSO-CMA

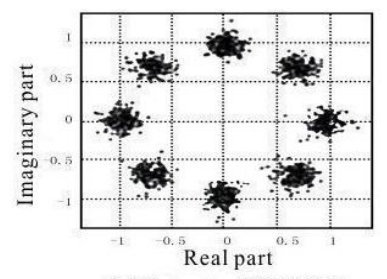

(b) Output of WT-CMA

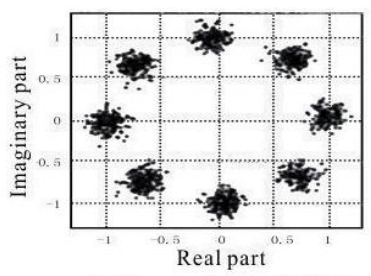

(d) Output of PSO-WTCMA

Figure 4. Simulation results

In Figure 4(a), the convergence of ACA-WT-CMA is the fastest, about 5000 steps faster than CMA, about 2500 steps faster than WT-CMA, about 1500 steps faster than ACA-CMA .After the convergence of these algorithms, the remaining symbols' interference are approximately the same, about $-22 \mathrm{~dB}$. Figure 4 (B, C, d) show that, output constellation can effectively disperse after the convergence of these algorithms. The output constellation of the ACA-WT-CMA is more clear and compact than ACA-CMA and WT-CMA .This shows that, ACA-WT-CMA has the fastest convergence speed, clear output constellation and smaller intersymbol interference for the QPSK signal.

Experiment 2: The two paths' phase of underwater acoustic channel is $\mathrm{C}=\left[\begin{array}{lll}-0.35 & 00 & 1\end{array}\right]$; he launch signal is 16QAM,the length of equalizer is $16, S N R$ is $20 \mathrm{~dB}$; the step lengths are $\mu_{C M A} 0.00001 、 \mu_{W T-C M A}=0.0002 、 \mu_{P S O-C M A}=0.00004 、 \mu_{P S O-W T-C M A}=0.00025$ respectively; in

CMA and WT-CMA, the fourth tap coefficient is set as 1, the remaining are 0; using DB2 orthogonal wavelet to decompose the input signal of the channel ,decomposition level-2 layer, 
forgetting factor $\beta=0.999$, initial power value is set as 4; results of 20 times Monte Kano simulation as shown in Figure 5.

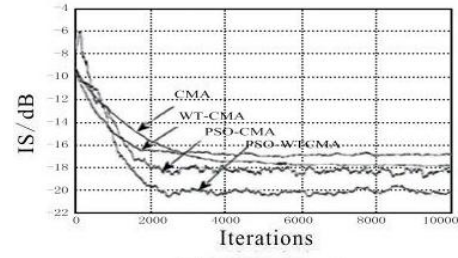

(a) Curves of ISI

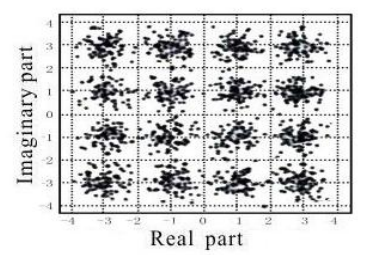

(c) Output of PSO-CMA

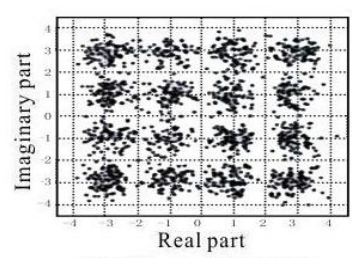

(b) Output of WT-CMA

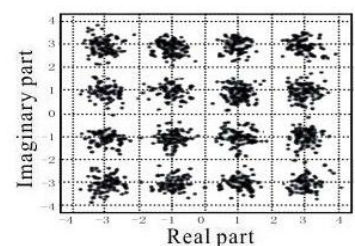

(d) Output of PSO-WTCMA

Figure 5. Simulation results

In Figure 5(a), the convergence of ACA-WT-CMA is the fastest, about 4500 steps faster than CMA, about 2500 steps faster than WT-CMA, about 1500 steps faster than ACA-CMA. After the convergence of these algorithms, the remaining symbols' interference are approximately the same, about -20dB.Figure 4 (b, c, d) show that, output constellation can effectively disperse after the convergence of these algorithms. The output constellation of the ACA-WT-CMA is more clear and compact than ACA-CMA and WT-CMA .This shows that, ACA-WT-CMA has the fastest convergence speed, clear output constellation and smaller intersymbol interference for the QPSK signal.

\section{Conclusion}

Based on the analysis of ACA, this paper combines CMA with ACA, uses ACA's character of searching optimum globally to initialize the weight vector of the equalizer, propose ACA-CMA. ACA-CMA is great, since CMA has slow convergence speed, large intersymbol interference, and WT-CMA is easy to fall into local convergence, difficult to obtain the global optimum. The simulation results of underwater acoustic channel show that ACA-WT-CMA is greatly improved in improving the convergence speed and reducing the intersymbol interference.

\section{References}

[1] R. S. Parpinelli, H. S. Lopes and A. A. Freitas, "Data mining with an ant colony optimization algorithm", IEEE transactions on Evolutionary Computing, vol. 6, no. 4, (2010), pp. 321-332.

[2] C. G. Di, D. M. Ant, "Net: Distributed stigmergetic control for communications networks", Journal of Artificial Intelligenee Researeh (JAIR), vol. 9, (2008), pp. 317-365.

[3] E. Lumer and B. Faieta, "Diversity and adaptation in Populations of clustering ants", Proc of the 3 Conf on Simulation of Adaptive Behavior, MIT Press, (2004), pp. 499-508.

[4] R. S. Parpinelli, H. S. Lopes and A. A. Freitas, "Data mining with an ant colony optimization algorithm", IEEE Transactions on Evolutionary Computation, vol. 6, no. 4, (2010), pp. 321-332.

[5] E. G. Talbi, O. Roux, C. FonluPt and D. Robillard, "Parallel ant colonies for the quadratic assignment problem”, Future Generation Computer Systems, vo. 17, no. 4, (2011), pp. 441-449. 
[6] L. M. De Campos, J. M. Fernndez Luna, J. Gumez and J. M. Apuerta, “Ant colony optimization for learning Bayesian networks", International Journal of Approximate Reasoning, vol. 31, no. 3, (2012), pp. 291-311.

[7] S. Li, J. T. Kwok and Y. Wang, "Using the discrete wavelet frame transform to merge landsat TM and spot panchromatic images", Information Fusion, vol. 3, no. 2, (2012), pp. 17-23.

[8] S. Mallat, "Singularity detection and processing with wavelet", IEEE Trans. Information Theory, vol. 38, no. 2, (1992), pp. 617-693.

[9] F. Pedersen, "A Gabor Expansion-based positive time-dependent power spectrum", IEEE Trans. Signal Processing, vol. 47, no. 2, (2009), pp. 589-590.

[10] R. F. Daniel, "A New transform for time-frequency analysis”, IEEE Trans. Signal Processing, vol. 40, no. 7, (2009), pp. 1697-1706.

[11] I. Daubechis, "the lectures on wavelets", Philadelphia, Society for Industrial and Applied Mathematics, (2008). 
International Journal of Control and Automation Vol.7, No.5 (2014) 